\title{
REDUCTION OF COMPLEXITY IN LEARNING DEXTEROUS MULTI-FINGERED MOTIONS: A THEORETICAL EXPLORATION INTO A FUTURE PROBLEM C. E. SHANNON RAISED*
}

\author{
SUGURU ARIMOTO ${ }^{\dagger}$
}

\begin{abstract}
This paper aims at unveiling the secret of the human ability for skilled motion that a multi-fingered hand with soft fingertips generates for executing a variety of dexterous tasks in his everyday life. As an elementary process of such dexterous motions, the dynamics of pinching an object by means of two multi-degrees of freedom robot fingers with soft and deformable tips are derived and analyzed. It is shown that passivity analysis leads to the effective design of a feedback control signal that realizes dynamic stable pinching, regardless of a complicated nonlinear structure of motion equation of the over-all system in which extra terms of Lagrange's multipliers arise from holonomic constraints of tight area-contacts between soft finger-tips and surfaces of the rigid object. It is then shown that a principle of linear superposition is applicable to the design of additional feedback signals for controlling both the posture (rotational angle) and the position (some of task coordinates of the mass center) of the object under the condition of stationary resolution of the controlled position-state variables. It is finally claimed that complexity of learning such an over-all skilled motion of pinching an object stably and controlling it at a prescribed posture and position can be drastically reduced from an exponential order to a linear order of the sum of complexities of learning each resolved motion separately, correspondingly to 1) stable pinching, 2) specification of the rotational angle for the object, and 3) that of some of position coordinates.
\end{abstract}

1. Introduction. It is not widely known in the robotics world but noticed among information theoretists enthusiastically admiring Shannon's whole works that Dr. C. E. Shannon, the founder father of information theory, was also extremely interested in robotics. According to C. E. Shannon Collected Papers [1], he built a maze-solving machine called "Theseus" in 1950, just a half century ago, which was a proto-type of maze-solving micro mice built by enough engineers for a variety of micro mouse maze contests over the world. He wrote a paper "Programming a Computer for Playing Chess" in 1950 [2], which bore fruit in 1997 when the IBM computer "Deep Blue" defeated the World Chess Champion. At the same time Shannon enjoyed constructing amusing gadgets and machines in his life, among which Mr. John Horgan, an interviewer from the Scientific American, recognized Shannon's seven chess-playing machines at his house (see page XVI in [1]). The author himself had the good fortune to see how one of his chess-playing machines with three fingers picked up, moved, and placed pieces in the screening of his $8 \mathrm{~mm}$ film that he brought to Kyoto, Japan, when in the year of 1985 he was awarded the first Kyoto Prize in the field of basic natural science. In 1983, Dr. Shannon wrote, "What can we expect in the future? Three advances in the artificial intelligence area would be most welcome. (1) An optical sensor-computer combination capable of learning to recognize objects, people, etc., as our eyes and occipital cortex do. (2) A manipulator-computer combination capable of the purposeful operations of the human hand. (3) A computer program capable of at least some of the concept formation and generalizing abilities of the human brain,

\footnotetext{
*Invited paper; received April 25, 2000; accepted for publication June 9, 2000.

${ }^{\dagger}$ Department of Robotics, Ritsumeikan University, Kusatsu, Shiga, 525-8577 Japan, TEL:+8177-561-3903, FAX:+81-77-561-2665, email: arimoto@se.ritsumei.ac.jp
} 
(see page XVI in [1])". Nevertheless, Dr. Shannon did not show any theoretical interrelations between the mathematical information theory and the design of his favorite brainchildren, though "Shannon himself suggests that applying information theory to biological systems may not be so farfetched, because in his view common principles underlie mechanical and living things" (see page XVII in [1]).

This paper attempts to challenge the problem how to explicate human ability of becoming skillful at pinching things by his thumb and fingers in mathematical terms (see Fig. 1). To do this, full dynamics of horizontal planar motion of a set of dual robot fingers pinching a rigid object via soft and deformable finger-tip material are derived by using Enler-Lagrange's formalism. By analyzing the dynamics from the viewpoint of input-output passivity for the overall system, we find two basic physical principles for synthesizing an overall feedback control signal that realizes a specified skilled motion. The first physical principle is called a "principle of superposition" of feedback signals (see Fig. 2), from which the overall feedback control signal realizing the specified skilled motion is generated as a linear sum of separate feedback signals designed independently such as 1) a feedback signal designed for realizing stable grasping, 2) for regulating the rotational angle of the object, and 3) or/and for positioning the object in cartesian coordinates. It should be remarked however that in order to apply the principle of superposition the closed-loop system must have a unique stationary resolution in such a sense that the target state of position variables can be decomposed into a combination of $f_{1}=f_{2}=f_{d}$ (corresponding to stable grasping with internal force $f_{d}$ ), $\theta=\theta_{d}$ (a desired rotation of the object), $x=x_{d}$ or $/$ and $y=y_{d}$ and a solution trajectory to the closed-loop differential system under geometric constraints of tight area-contacts converges asymptotically to this unique target position-state. It is shown in more detail that if a set of chosen Jacobian vectors associated with those feedback signals $\Delta f_{i}\left(=f_{i}-f_{d}\right), \Delta \theta\left(=\theta-\theta_{d}\right), \Delta x\left(=x-x_{d}\right)$ or/and $\Delta y\left(=y-y_{d}\right)$ are linearly independent then LaSalle's invariance theorem assures the unique stationary resolution. This finding can be interpreted that complexity of learning the overall skilled motion can be reduced drastically from exponential order of $N_{f} \times N_{\theta} \times N_{x}$ to linear order of $N_{f}+N_{\theta}+N_{x}$, a linear sum of complexities corresponding to learning each motion separately, where $N_{f}$ (or $N_{\theta}, N_{x}$ ) may stand for a number of practices needed to acquire the ability of grasping stably an object (or that of regulating the rotation of the object, that of positioning).

2. Dynamics of Pinching Motion. Firstly let us derive the dynamics of pinch motion by means of dual fingers with soft tips (see Fig.3). There are four equations of geometric constraint as shown in the following:

$$
\begin{aligned}
& Y_{1}=c_{1}-r_{1} \varphi_{1}=c_{1}-r_{1}\left(\pi+\theta-q_{11}-q_{12}\right) \\
& Y_{2}=c_{2}-r_{2} \varphi_{2}=c_{2}-r_{2}\left(\pi-\theta-q_{21}-q_{22}\right)
\end{aligned}
$$




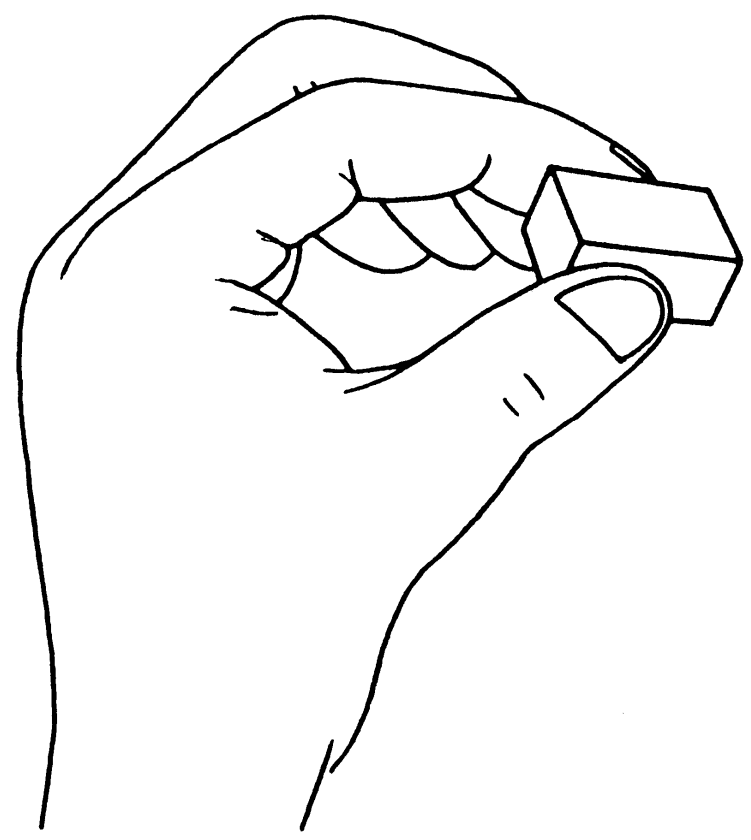

FIG. 1. Motion of pinching an object

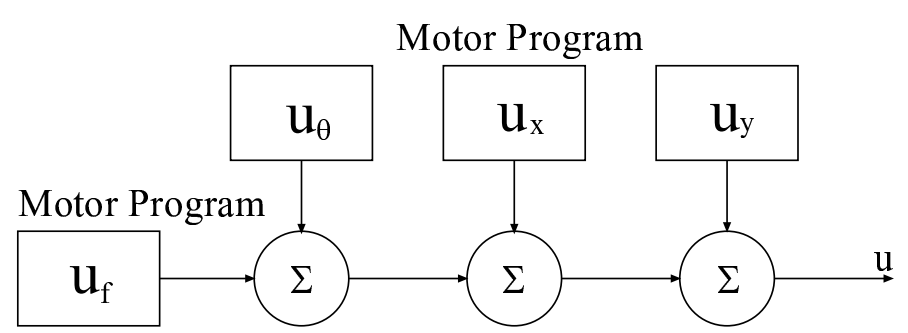

FIG. 2. The principle of Superposition

$$
\begin{aligned}
x & =x_{1}+\frac{l}{2} \cos \theta-Y_{1} \sin \theta \\
& =x_{2}-\frac{l}{2} \cos \theta-Y_{2} \sin \theta \\
y & =y_{1}-\frac{l}{2} \sin \theta-Y_{1} \cos \theta \\
& =y_{2}+\frac{l}{2} \sin \theta-Y_{2} \cos \theta
\end{aligned}
$$

where all symbols are defined in Fig.3. The first two equations arise from tight areacontacts between each surface of finger-tips and the surface of the rigid object. The left hand sides of eqs.(1) and (2) should be calculated by the half of state variables, that is, position variables $z=(x, y, \theta)^{\mathrm{T}}, q_{1}=\left(q_{11}, q_{12}\right)^{\mathrm{T}}$, and $q_{2}=\left(q_{21}, q_{22}\right)^{\mathrm{T}}$ as 
follows :

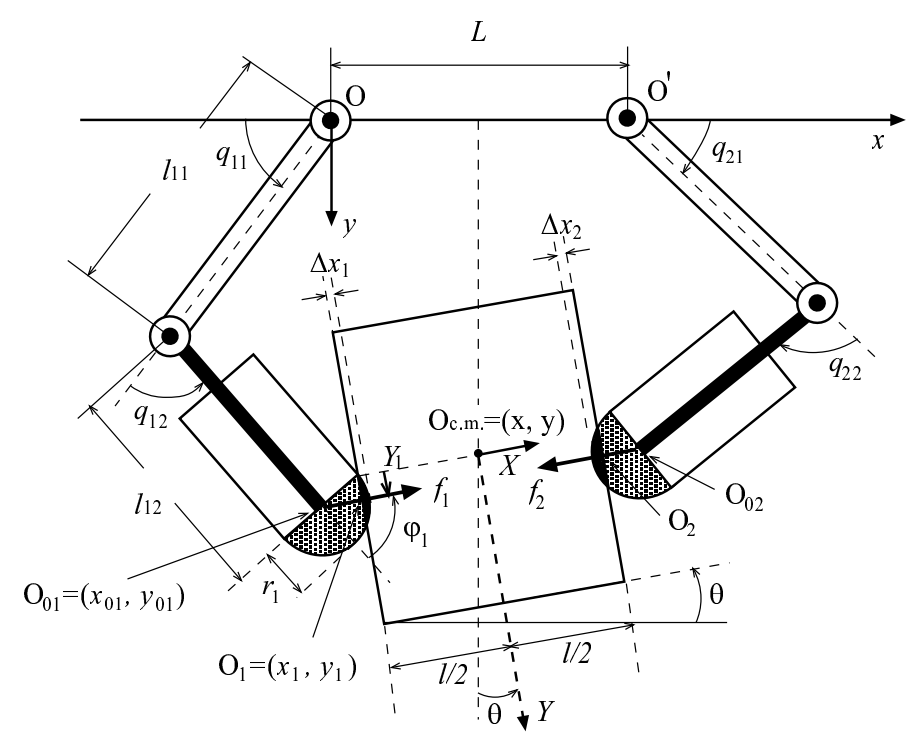

FIG. 3. A setup of dual fingers with soft tips pinching a rigid object

$$
\begin{aligned}
& Y_{1}=\left(x_{01}-x\right) \sin \theta+\left(y_{01}-y\right) \cos \theta \\
& Y_{2}=\left(x_{02}-x\right) \sin \theta+\left(y_{02}-y\right) \cos \theta
\end{aligned}
$$

where

$$
\begin{aligned}
& x_{01}=-l_{11} \cos q_{11}-l_{12} \cos \left(q_{11}+q_{12}\right) \\
& y_{01}=l_{11} \sin q_{11}+l_{12} \sin \left(q_{11}+q_{12}\right) \\
& x_{02}=L+l_{21} \cos q_{21}+l_{22} \cos \left(q_{21}+q_{22}\right) \\
& y_{02}=l_{21} \sin q_{21}+l_{22} \sin \left(q_{21}+q_{22}\right)
\end{aligned}
$$

The second two equations (3) and (4) follow from the fact that the loop starting from the origin $O$ of the first joint center of the left finger and returning to it through $O_{01}$, $O_{1}, O_{c . m}, O_{2}, O_{02}$ and $O^{\prime}$ is closed (see Fig.3). Then, it is reasonable to introduce Lagrange's multipliers $\lambda_{1}, \lambda_{2}, \lambda_{x}$ and $\lambda_{y}$ for corresponding constraint equations (1) to (4) and consider the two quantities

$$
\begin{aligned}
Q= & \lambda_{x}\left\{\left(x_{1}-x_{2}\right)+l \cos \theta-\left(Y_{1}-Y_{2}\right) \sin \theta\right\} \\
& +\lambda_{y}\left\{\left(y_{1}-y_{2}\right)-l \sin \theta-\left(Y_{1}-Y_{2}\right) \cos \theta\right\} \\
S= & \sum_{i=1,2} \lambda_{i}\left(Y_{i}-c_{i}+r_{i} \varphi_{i}\right)
\end{aligned}
$$

On the other hand, the distributed pressure arised from the deformation of each fingertip can be lumped-parametrized into the single reproducing force $f_{i}\left(\Delta x_{i}\right)=K_{i} \Delta x_{i}^{2}$ for $i=1,2$, as discussed in appendix of the previous paper [3], which has direction $\pm(\cos \theta,-\sin \theta)^{\mathrm{T}}$ as shown in Fig. 3 . 
It has been also shown in [3] that both the gradients of $Q$ with respect to $\left(x_{0 i}, y_{0 i}\right)$ for $i=1,2$ arise in the same direction as the lumped-parameterized reproducing force $f_{i}\left(\Delta x_{i}\right)$ (that is, $\left.(\cos \theta,-\sin \theta)^{\mathrm{T}}\right)$ and hence the effects of this constraint force on Lagrange's equation of the overall sysytem should be merged in $f_{i}\left(\Delta x_{i}\right)$ appearing in it. Thus, the Lagrangian of the overall system can be given as

$$
L=K-P+S
$$

where $S$ is defined by eq.(11) and $K$ and $P$ signify the kinetic energy and potential energy respectively defined as

$$
\begin{aligned}
K & =\sum_{i=1,2} \frac{1}{2} \dot{q}_{i}^{\mathrm{T}} H_{i}\left(q_{i}\right) \dot{q}_{i}+\frac{1}{2} \dot{z}^{\mathrm{T}} H \dot{z} \\
P & =\sum_{i=1,2} \int_{0}^{\Delta x_{i}} f_{i}(\xi) \mathrm{d} \xi
\end{aligned}
$$

where $H=\operatorname{diag}(M, M, I), M$ and $I$ denote the mass and inertia moment of the rigid object, and $\Delta x_{i}$ denotes the maximum displacement of deformation for $i=1,2$. Applying Hamilton's principle for the variational form

$$
\int_{t_{1}}^{t_{2}}\left\{\delta(K-P+S)+u_{1}^{\mathrm{T}} \delta q_{1}+u_{2}^{\mathrm{T}} \delta q_{2}\right\} \mathrm{d} t=0
$$

we obtain the Lagrange equation of the overall system descibed as follows (see [3] and [4])

$$
\begin{gathered}
\left\{H_{i}\left(q_{i}\right) \frac{\mathrm{d}}{\mathrm{d} t}+\frac{1}{2} \dot{H}_{i}\left(q_{i}\right)\right\} \dot{q}_{i}+S_{i}\left(q_{i}, \dot{q}_{i}\right) \dot{q}_{i}+(-1)^{i-1} J_{0 i}^{\mathrm{T}}\left(\begin{array}{c}
\cos \theta \\
-\sin \theta
\end{array}\right) f_{i} \\
+\lambda_{i}\left\{r_{i}\left(\begin{array}{c}
1 \\
1
\end{array}\right)-J_{0 i}^{\mathrm{T}}\left(\begin{array}{c}
\sin \theta \\
\cos \theta
\end{array}\right)\right\}=u_{i} \quad(i=1,2) \\
H \ddot{z}+\sum_{i=1,2}(-1)^{i}\left(\begin{array}{c}
\cos \theta \\
-\sin \theta \\
Y_{i}
\end{array}\right) f_{i}\left(\Delta x_{i}\right) \\
+\sum_{i=1,2} \lambda_{i}\left(\begin{array}{c}
\sin \theta \\
\cos \theta \\
(-1)^{i}\left(\Delta x_{i}-\frac{1}{2} l\right)
\end{array}\right)=0
\end{gathered}
$$

Here, eq.(16) denotes dynamics of the left finger for $i=1$ and the right one for $i=2$ and eq.(17) denotes dynamics of the rigid object. As is shown in [3], the overall dynamics of eqs.(16) and (17) satisfy passivity, that is, satisfy the relation:

$$
\int_{0}^{t}\left(\dot{q}_{1}^{\mathrm{T}} u_{1}+\dot{q}_{2}^{\mathrm{T}} u_{2}\right) \mathrm{d} \tau=E(t)-E(0) \geq-E(0)
$$

where $E$ signifies the total energy of the overall system, that is,

$$
E=K+P
$$

Symbols $J_{0 i}$ for $i=1,2$ appearing in (16) denote Jacobian matrices of $\left(x_{0 i}, y_{0 i}\right)^{\mathrm{T}}$ with respect to $\left(q_{i 1}, q_{i 2}\right)^{\mathrm{T}}$ for $i=1,2$ respectively. 
3. Design of Feedback Signals. Given a desired internal force $f_{d}$ and rotational angle $\theta_{d}$, consider the feedback control signal

$$
\begin{aligned}
& u_{f i}=-k_{v i} \dot{q}_{i}+(-1)^{i-1} f_{d}\left\{J_{0 i}^{\mathrm{T}}\left(\begin{array}{c}
\cos \theta \\
-\sin \theta
\end{array}\right)-\frac{r_{i}}{r_{1}+r_{2}}\left(Y_{1}-Y_{2}\right)\left(\begin{array}{c}
1 \\
1
\end{array}\right)\right\} \\
& u_{\theta i}=(-1)^{i} \zeta^{-1}(\beta \Delta \theta+\alpha \dot{\theta})\left\{J_{0 i}^{\mathrm{T}}\left(\begin{array}{c}
\sin \theta \\
\cos \theta
\end{array}\right)-r_{i}\left(\begin{array}{c}
1 \\
1
\end{array}\right)\right\}
\end{aligned}
$$

where $\quad \Delta \theta=\theta-\theta_{d}, \quad \beta>0, \alpha \geq 0$ and

$$
\zeta=\left(l-\Delta x_{1}-\Delta x_{2}\right)
$$

As shown in [3], $u_{f i}$ is designed for realizing dynamic stable grasping and $u_{\theta_{i}}$ is designed for regulating the rotational angle $\theta$ to the target $\theta=\theta_{d}$. Substituting $u_{i}=u_{f_{i}}+u_{\theta_{i}}+\Delta u_{i}$ into eq.(16) yields

$$
\begin{array}{r}
\left\{H_{i}\left(q_{i}\right) \frac{\mathrm{d}}{\mathrm{d} t}+\frac{1}{2} \dot{H}_{i}\left(q_{i}\right)\right\} \dot{q}_{i}+\left\{S_{i}\left(q_{i}, \dot{q}_{i}\right)+k_{v i} I\right\} \dot{q}_{i}+(-1)^{i-1}\left\{J_{0 i}^{\mathrm{T}}\left(\begin{array}{c}
\cos \theta \\
-\sin \theta
\end{array}\right) \Delta f_{i}\right. \\
\left.+\frac{r_{i} f_{d}}{r_{1}+r_{2}}\left(Y_{1}-Y_{2}\right)\left(\begin{array}{c}
1 \\
1
\end{array}\right)\right\}+\lambda_{i}\left\{r_{i}\left(\begin{array}{c}
1 \\
1
\end{array}\right)-J_{0 i}^{\mathrm{T}}\left(\begin{array}{c}
\sin \theta \\
\cos \theta
\end{array}\right)\right\}-u_{i \theta}=\Delta u_{i}
\end{array}
$$

At the same time it is important to rewrite the equation (17) into

$$
\begin{aligned}
M \ddot{x}-\left(\Delta f_{1}-\Delta f_{2}\right) \cos \theta+\left(\lambda_{1}+\lambda_{2}\right) \sin \theta & =0 \\
M \ddot{y}+\left(\Delta f_{1}-\Delta f_{2}\right) \sin \theta+\left(\lambda_{1}+\lambda_{2}\right) \cos \theta & =0 \\
I \ddot{\theta}-\Delta f_{1} Y_{1}+\Delta f_{2} Y_{2}-f_{d}\left(Y_{1}-Y_{2}\right)-\lambda_{1} \Delta x_{1}+\lambda_{2} \Delta x_{2} & =-\frac{l}{2}\left(\lambda_{1}-\lambda_{2}\right)
\end{aligned}
$$

By the same way as given in the previous paper [3] it is possible to show that the input-output pair $\left\{\left(\Delta u_{1}, \Delta u_{2}\right),\left(\dot{q}_{1}, \dot{q}_{2}\right)\right\}$ of eqs.(23) to (26) satisfies passivity. More precisely, we obtain

$$
\begin{gathered}
\int_{0}^{t}\left(\dot{q}_{1}^{\mathrm{T}} \Delta u_{1}+\dot{q}_{2}^{\mathrm{T}} \Delta u_{2}\right) \mathrm{d} \tau= \\
W(t)-W(0)+\int_{0}^{t}\left\{k_{v 1}\left\|\dot{q}_{1}\right\|^{2}+k_{v 2}\left\|\dot{q}_{2}\right\|^{2}+\alpha \dot{\theta}^{2}\right\} \mathrm{d} \tau
\end{gathered}
$$

where $W$ is defined as

$$
W=K+\Delta P+\frac{f_{d}}{2\left(r_{1}+r_{2}\right)}\left(Y_{1}-Y_{2}\right)^{2}+\frac{\beta}{2} \Delta \theta^{2}
$$

where $\delta x_{i}=\Delta x_{i}-\Delta x_{d i}$ and $\Delta x_{d i}=f_{i}^{-1}\left(f_{d}\right)$, and

$$
\Delta P=\sum_{i=1,2} \int_{0}^{\delta x_{i}}\left\{f_{i}\left(\xi+\Delta x_{d i}\right)-f_{d}\right\} \mathrm{d} \xi
$$

Now note that the dimension of the state space of the dynamics of eqs.(23) to (26) is 14 since there are position state variables $q_{1}, q_{2}$ and $z$ and velocity state variables 
$\dot{q}_{1}, \dot{q}_{2}$ and $\dot{z}$. However, the DOF of the overall system is not 7 but 5 because the motion is subject to geometric constraints described by eqs.(1) and (2). Hence, the set of all states defined by $S G=\left\{\left(q_{1}, q_{2}, z, \dot{q}_{1}, \dot{q}_{2}, \dot{z}\right): \dot{q}_{1}=0, \dot{q}_{2}=0, \dot{z}=0, \delta x_{1}=\right.$ $\delta x_{2}=0, Y_{1}-Y_{2}=0, \Delta \theta=0$, together with constraints of eqs.(1) and (2) $\}$ becomes of 1-dimension in the 14-dimensional state space. It is also important to note that the scalar function $W$ can not be a Lyapunov function for the system of eqs.(23) to (26) with $\Delta u_{i}=0$ for $i=1,2$ even if its time derivative $\dot{W}$ is non-positive definite, $\mathrm{i}, \mathrm{e}$,

$$
\dot{W}(t)=-\sum_{i=1}^{2} k_{v_{i}}\left\|\dot{q}_{i}\right\|^{2}-\alpha \dot{\theta}^{2}
$$

because $W$ is not positive definite in the manifold defined by

$$
M=\left\{\left(q_{1}, q_{2}, z, \dot{q}_{1}, \dot{q}_{2}, \dot{z}\right): \text { under constraint eqs.(1) and (2) }\right\}
$$

Therefore, it is not possible to apply LaSalle's invariance Theorem [5] for proving

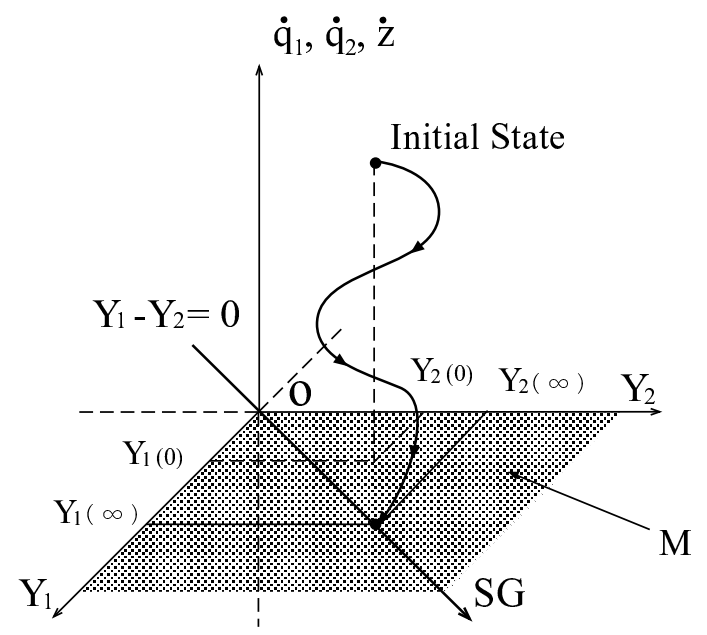

FIG. 4. A solution to the closed-loop equation converges asymptotically to the 1-dim. stable manifold $S G$. Here, $M$ denotes the hyperplane defined by $\dot{q}_{1}=0, \dot{q}_{2}=0$ and $\dot{z}=0$.

the asymptotic convergence of the solution to eqs.(23) to (26) to the 1-dimentional manifold SG.

Now, we assume that at the initial time $t=0$ the object is in the state of being pinched and magnitudes of all velocity vectors $\dot{q}_{1}(0), \dot{q}_{2}(0)$ and $\dot{z}(0)$ are not so large. In particular, the value of $W$ is small enough satisfying

$$
W(0)<\int_{0}^{\Delta x_{d_{i}}} f_{i}(\xi) \mathrm{d} \xi, \quad i=1,2
$$

Then, it follows that $\Delta P(t) \leq W(t) \leq W(0)$ and thereby $\Delta x_{i}$ for $i=1,2$ are positive. This implies that both fingers maintain contacting the object. Moreover, it is possible 
to check by taking velocity feedback gains $k_{v_{i}}$ for $i=1,2$ appropriately that $q_{i}(t)$ does not deviate much from $q_{i}(0)$ for $i=1,2$, because

$$
\lambda_{m_{i}}\left\|\dot{q}_{i}(t)\right\|^{2} \leq \dot{q}_{i}^{\mathrm{T}} H_{i}\left(q_{i}\right) \dot{q}_{i} \leq 2 W(t) \leq 2 W(0)-2 \int_{0}^{t} k_{v_{i}}\left\|\dot{q}_{i}(\tau)\right\|^{2} \mathrm{~d} \tau
$$

which means

$$
\left\|\dot{q}_{i}(t)\right\| \leq \sqrt{\frac{2 W(0)}{\lambda_{m_{i}}}}, \quad \int_{0}^{\infty}\left\|\dot{q}_{i}(t)\right\|^{2} \mathrm{~d} t \leq \frac{W(0)}{k v_{i}}
$$

where $\lambda_{m_{i}}$ denotes the minimum eigenvalue of $H_{i}\left(q_{i}\right)$ for all $q_{i}$. Hence, $\dot{q}_{i}(t) \in$ $L^{2}(0, \infty)$ for $i=1,2$ and $\left\|\dot{q}_{i}(t)\right\|$ for $i=1,2$ are uniformly bounded. Then, it is easy to check that all state variables $\left(q_{1}(t), q_{2}(t), z(t), \dot{q}_{1}(t), \dot{q}_{2}(t), \dot{z}(t)\right)$ remain in a neighborhood of its initial state in the 14-dimentional space. Next it is improtant to note that $\lambda_{1}$ and $\lambda_{2}$ can be expressed in terms of state variables by using constraint eqs.(1) and (2) and their first and second derivatives in time (see Appendix B). Thus, all state variables are uniformly bounded and $\lambda_{1}$ and $\lambda_{2}$ are also uniformly bounded. Then, according to eqs.(23) to (26), acceleration variables ( $\left.\ddot{q}_{1}, \ddot{q}_{2}, \ddot{z}\right)$ become uniformly bounded and thereby $\left(\dot{q}_{1}, \dot{q}_{2}, \dot{z}\right)$ become uniformly continuous. In particular, uniform continuity of $\dot{q}_{i}(t)$ together with the fact that $\dot{q}_{i}(t) \in L^{2}(0, \infty)$ implies

$$
\dot{q}_{i}(t) \rightarrow 0 \quad \text { as } t \rightarrow \infty \quad \text { for } \quad i=1,2
$$

Further, referring to the uniform continuity of $\ddot{q}_{i}$ and the convergence of eq.(35), we can conclude that $\ddot{q}_{i}(t) \rightarrow 0$ as $t \rightarrow \infty$ for $i=1,2$, too. On the other hand, eq.(A-1) shows(see Appendix A)that $\dot{\theta}(t) \rightarrow 0$ as $t \rightarrow 0$ because all $\dot{x}_{0 i}$ and $\dot{y}_{0 i}$ vanish as $t \rightarrow \infty$. This means that $\ddot{\theta}(t) \rightarrow 0$ as $t \rightarrow \infty$, because $\ddot{\theta}(t)$ is also uniformly continuous. Then, from eqs.(1) and (2) it follows that $\dot{Y}_{1} \rightarrow 0$ and $\dot{Y}_{2} \rightarrow 0$ as $t \rightarrow \infty$. Finally it is important to note that differentiating eqs.(5) and (6) in $t$ yields

$$
\dot{x} \sin \theta+\dot{y} \cos \theta \rightarrow 0 \text { as } t \rightarrow \infty
$$

and subsequently

$$
\ddot{x} \sin \theta+\ddot{y} \cos \theta \rightarrow 0 \text { as } t \rightarrow \infty
$$

since $\ddot{\theta} \rightarrow 0$ as $t \rightarrow \infty$. On the other hand, eqs.(24) and (25) are reduced to

$$
\ddot{x} \sin \theta+\ddot{y} \cos \theta+\lambda_{1}+\lambda_{2}=0
$$

which concludes that $\lambda_{1}+\lambda_{2} \rightarrow 0$ as $t \rightarrow \infty$.

Since $\lambda_{1}+\lambda_{2}$ and the angular velocity vectors $\dot{q}_{1}, \dot{q}_{2}, \dot{\theta}$ together with $\ddot{q}_{1}, \ddot{q}_{2}, \ddot{\theta}$ tend to vanish as $t \rightarrow \infty$, the remaining terms of eq.(23) with $\Delta u_{i}=0$ except the inertia and centripetal-Coriolis force terms can be expressed as follows:

$$
A r \rightarrow 0 \quad \text { as } \quad t \rightarrow \infty
$$

where

$$
A=\left[\begin{array}{cccc}
r_{1}\left(\begin{array}{l}
1 \\
1
\end{array}\right) & -J_{01}^{\mathrm{T}}\left(\begin{array}{c}
\sin \theta \\
\cos \theta
\end{array}\right) & J_{01}^{\mathrm{T}}\left(\begin{array}{r}
\cos \theta \\
-\sin \theta
\end{array}\right) & \left(\begin{array}{c}
0 \\
0
\end{array}\right) \\
-r_{2}\left(\begin{array}{l}
1 \\
1
\end{array}\right) & J_{02}^{\mathrm{T}}\left(\begin{array}{l}
\sin \theta \\
\cos \theta
\end{array}\right) & \left(\begin{array}{l}
0 \\
0
\end{array}\right) & -J_{02}^{\mathrm{T}}\left(\begin{array}{r}
\cos \theta \\
-\sin \theta
\end{array}\right)
\end{array}\right]
$$




$$
r=\left[\begin{array}{r}
\lambda_{1}+\frac{f_{d}}{r_{1}+r_{2}}\left(Y_{1}-Y_{2}\right)-\beta \zeta^{-1} \Delta \theta \\
\lambda_{1}-\beta \zeta^{-1} \Delta \theta \\
\Delta f_{1} \\
\Delta f_{2}
\end{array}\right]
$$

It should be noted that the $4 \times 4$-matrix $A$ is nonsingular. Thus, it follows that $r \rightarrow 0$ as $t \rightarrow \infty$. Hence, from the last two elements of $r$ defined by (41) it follows that $\Delta f_{1} \rightarrow 0$ and $\Delta f_{2} \rightarrow 0$ as $t \rightarrow \infty$. Then, by subtracting the second component of $r$ from the first one of $r$, we obtain

$$
Y_{1}-Y_{2} \rightarrow 0 \quad \text { as } \quad t \rightarrow \infty
$$

On the other hand, note that it follows from eq.(26) that

$$
\left(l-\Delta x_{1}-\Delta x_{2}\right) \lambda_{1}-f_{i}\left(Y_{1}-Y_{2}\right) \rightarrow 0 \quad \text { as } \quad t \rightarrow \infty
$$

Substituting eq.(42) into this yields $\lambda_{1} \rightarrow 0$ as $t \rightarrow \infty$. Then, from the second component of $r$ it follows that $\Delta \theta \rightarrow 0$ as $t \rightarrow \infty$. In this way, we could prove the following theorem:

Theorem 1 For given $f_{d}>0$ and $\theta_{d}>0$, the state vector of the solution to the closed-loop system of eqs.(23) to (26) with $\Delta u_{1}=0$ and $\Delta u_{2}=0$ converges asymptotically to the 1-dimensional manifold SG of dynamic stable grasping (see Fig.4), provided that inequality (32) is satisfied at $t=0$.

4. Principle of Superposition. According to Theorem 1, the skilled motion composed of dynamic stable grasping (pinching) and regulation of rotation angle of the object is realized by the overall control input signal $u_{i}=u_{f_{i}}+u_{\theta_{i}} \quad(i=1,2)$. It should be remarked at this stage that a single control input $u_{f_{i}}(i=1,2)$ itself realizes stable grasping in general. In fact, if $u_{i}=u_{f_{i}}(i=1,2)$ in eq.(16), then the closed-loop system satisfies the following relation:

$$
\frac{\mathrm{d}}{\mathrm{d} t} V(t)=-\int_{0}^{t} \sum_{i=1,2} k_{v_{i}}\left\|\dot{q}_{i}\right\|^{2} \mathrm{~d} t
$$

where

$$
V=K+\Delta P+\frac{f_{d}}{2\left(r_{1}+r_{2}\right)}\left(Y_{1}-Y_{2}\right)^{2}
$$

This means that a solution to the closed-loop system of eqs.(16) and (17) with $u_{i}=$ $u_{f_{i}} \quad(i=1,2)$ converges asymptiotically to the 2 -dimensional manifold

$$
\begin{aligned}
& S G_{f}=\left\{\left(q_{1}, q_{2}, z, \dot{q}_{1}=0, \dot{q}_{2}=0, \dot{z}=0\right):\right. \\
&\left.f_{1}\left(\Delta x_{1}\right)=f_{2}\left(\Delta x_{2}\right)=f_{d}, Y_{1}=Y_{2}, \text { eqs.(1) and }(2)\right\}
\end{aligned}
$$

This is an extended result of Nguyen's reasoning (see [6]) of stable grasping from the static viewpoint to the dynamic one. Then, it is reasonable to say that the pair $\left\{u_{f_{i}}, \dot{q}_{i}\right.$ for $\left.i=1,2\right\}$ constitutes a conjugate power pair. Now consider again the input 
signal $v_{\theta_{i}}, i=1,2$, for regulating rotational angle of the object in addition to $u_{f_{i}}$, $i=1,2$. Then, the closed-loop system of eqs.(23) to (26) with $\Delta u_{i}=0$ satisfies

$$
\int_{0}^{t} \sum_{i=1,2}-\dot{q}_{i}^{\mathrm{T}} u_{\theta_{i}} \mathrm{~d} \tau=\Delta V(t)-\Delta V(0)+\int_{0}^{t} \alpha \dot{\theta}^{2} \mathrm{~d} \tau
$$

where

$$
\Delta V=W-V=\frac{\beta}{2} \Delta \theta^{2}
$$

Note that $\Delta V$ is non-negative definite and the right hand side of eq.(47) is bounded from below. We say that such input $u_{\theta_{i}}(i=1,2)$ together with $\dot{q}_{i}(i=1,2)$ constitutes a conjugate power pair posterior to the stable grasping control input $u_{f_{i}}(i=1,2)$. Thus, it is possible to see the linear superposition of $u_{\theta_{i}}(i=1,2)$ on $u_{f_{i}}$ constitutes the conjugate power pair together with $\dot{q}_{i}(i=1,2)$. It is then important to note that according to Theorem 1 the solution trajectory to the closed-loop system of eqs.(23) to (26) with $\Delta u_{i}=0$ converges to the 1-dim. manifold SG (see Fig.4). This can be interpreted that the half state of position variables is resolved into the stationary state $\left\{f_{1}=f_{2}=f_{d}, Y_{1}=Y_{2}(=\eta), \theta=\theta_{d}\right\}$ which, together with constraints of eqs.(1) and (2), characterizes the manifold SG. We call such convergence to the 1-dimensional manifold SG the stationary resolution of position state variables. Next, consider the problem to regulate position of the mass center of the object in x-axis or y-axis, since the manifold SG is of 1-dimension and parametrized by $\eta\left(=Y_{1}=Y_{2}\right)$, which can be used for determination of the value of $x$ or $y$ in the cartesian coordinates of the object mass center $O_{c . m .}=(x, y)$. To show this, we assume for simplicity that $r_{1}=r_{2}=r$

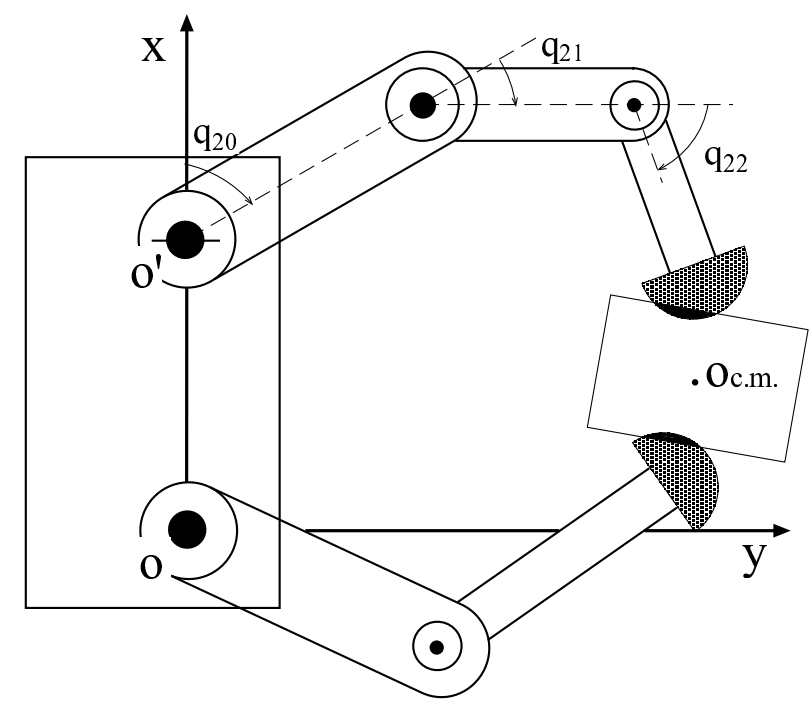

FIG. 5. Pinching by a pair of thumb (2DOF) and index (3DOF)

and characteristics of reproducing forces of both the soft finger-tips in maximum displacements are the same, i.e., $f_{1}(\Delta x)=f_{2}(\Delta x)$. Then, for a given desired position 
$x_{d}$ in $\mathrm{x}$-direction, consider the input signal

$$
u_{x i}=\gamma\left[r \sin \theta_{d}\left(\begin{array}{c}
1 \\
1
\end{array}\right)-\left(\begin{array}{c}
\frac{\partial x_{0 i}}{\partial q_{i 1}} \\
\frac{\partial x_{0 i}}{\partial q_{i 2}}
\end{array}\right)\right]\left(x^{\prime}-x_{d}\right), \quad i=1,2
$$

where

$$
x^{\prime}=\frac{x_{01}+x_{02}}{2}-\frac{Y_{1}+Y_{2}}{2} \sin \theta_{d}
$$

Then, it is easy to see that

$$
-\int_{0}^{t} \sum_{i=1,2} \dot{q}_{i}^{\mathrm{T}} u_{x_{i}} \mathrm{~d} \tau=\frac{\gamma}{2}\left(x^{\prime}-x_{d}\right)^{2}
$$

Note that from eqs.(3), (4), and (A-7) and (A-8) of relations between $x_{i}$ and $x_{0 i}$ $(i=1,2)$ it follows that

$$
x=x^{\prime}-\frac{1}{2}\left(\Delta x_{1}-\Delta x_{2}\right) \cos \theta-\frac{Y_{1}+Y_{2}}{2}\left(\sin \theta-\sin \theta_{d}\right)
$$

This means that $\left(u_{x_{1}}, u_{x_{2}}\right)$ together with $\left(\dot{q}_{1}, \dot{q}_{2}\right)$ constitutes the conjugate power pair posterior to the input $u_{f_{i}}+u_{\theta_{i}}, i=1,2$. Since $x \rightarrow x^{\prime}$ where $\Delta x_{1}-\Delta x_{2} \rightarrow 0$ and $\Delta \theta \rightarrow 0$, we are now able to state:

Theorem 2 The feedback control signal $u_{i}=u_{f_{i}}+u_{\theta_{i}}+u_{x_{i}}, i=1,2$, for eqs.(23) to (26) renders the trajectory of a solution to the closed-loop system convergent asymptotically to the $\mathrm{SG}_{0}=\left\{\left(q_{1}, q_{2}, z, \dot{q}_{1}=0, \dot{q}_{2}=0, \dot{z}=0\right): Y_{1}=Y_{2}, f\left(\Delta x_{1}\right)=\right.$ $f\left(\Delta x_{2}\right)=f_{d}, \theta=\theta_{d}, x=x_{d}$ and eqs.(1),(2)\}, provided that its initial state lies in some neighborhood of $\mathrm{SG}_{0}$ in 14-dimensional state space. It is interesting to note that the overall feedback control can be designed by linear superposition of feedback signals $u_{f i}, u_{\theta i}$ and $u_{x i}$ though dynamics of the relevant motion are nonlinear and subject to geometric constraints. It should be remarked that in this case the following relation holds :

$$
\frac{\mathrm{d}}{\mathrm{d} t} E=-\left\{k_{v_{1}}\left\|\dot{q}_{1}\right\|^{2}+k_{v_{2}}\left\|\dot{q}_{2}\right\|^{2}\right\}-\alpha \dot{\theta}^{2}
$$

where

$$
E=W+\frac{\gamma}{2}\left(x^{\prime}-x_{d}\right)^{2}
$$

Note that $E$ is now positive definite in the 10-dimensional manifold constrained by the eqs.(1) and (2) and their time derivatives. Then, it is possible to apply LaSalle's invariance theorem [4] and conclude that $\dot{q}$ and $\ddot{q}$ tend to vanish as $t \rightarrow \infty$, where $q=\left(q_{1}^{\mathrm{T}}, q_{2}^{\mathrm{T}}, z^{\mathrm{T}}\right)^{\mathrm{T}}$. Then, from eqs. $(24)$ and (26) it follows that

$$
\left(\begin{array}{cc}
-\cos \theta & \sin \theta \\
\sin \theta & \cos \theta
\end{array}\right)\left(\begin{array}{c}
\Delta f_{1}-\Delta f_{2} \\
\lambda_{1}+\lambda_{2}
\end{array}\right) \rightarrow\left(\begin{array}{l}
0 \\
0
\end{array}\right)
$$


as $t \rightarrow \infty$, This means that $\Delta f_{1}-\Delta f_{2} \rightarrow 0$ and $\lambda_{1}+\lambda_{2} \rightarrow 0$ as $t \rightarrow \infty$. Then, the remaining terms of eq.(23) except the inertia and centripetal-Coriolis terms should tend to vanish as $t \rightarrow \infty$, which is expressed by the form

$$
A v \rightarrow 0 \quad \text { as } \quad t \rightarrow \infty
$$

where

$A=\left[\begin{array}{rrrr}r\left(\begin{array}{l}1 \\ 1\end{array}\right) & -J_{01}^{\mathrm{T}}\left(\begin{array}{c}\sin \theta \\ \cos \theta\end{array}\right) & J_{01}^{\mathrm{T}}\left(\begin{array}{r}\cos \theta \\ -\sin \theta\end{array}\right) & r \sin \theta_{d}\left(\begin{array}{l}1 \\ 1\end{array}\right)-\left(\frac{\partial x_{01}}{\partial q_{1}}\right)^{\mathrm{T}} \\ -r\left(\begin{array}{l}1 \\ 1\end{array}\right) & J_{02}^{\mathrm{T}}\left(\begin{array}{c}\sin \theta \\ \cos \theta\end{array}\right) & -J_{02}^{\mathrm{T}}\left(\begin{array}{r}\cos \theta \\ -\sin \theta\end{array}\right) & r \sin \theta_{d}\left(\begin{array}{l}1 \\ 1\end{array}\right)-\left(\frac{\partial x_{02}}{\partial q_{2}}\right)^{\mathrm{T}}\end{array}\right]$

$$
v=\left[\begin{array}{r}
\lambda_{1}+\frac{f_{d}}{2 r}\left(Y_{1}-Y_{2}\right)-\beta \zeta^{-1} \Delta \theta \\
\lambda_{1}-\beta \zeta^{-1} \Delta \theta \\
\Delta f_{1} \\
\gamma\left(x^{\prime}-x_{d}\right)
\end{array}\right]
$$

It is easy to check that the matrix $A$ is of full rank and hence $v \rightarrow 0$ as $t \rightarrow \infty$. This means that $\Delta f_{1} \rightarrow 0, x^{\prime}-x_{d} \rightarrow 0, \Delta f_{2} \rightarrow 0$ as $t \rightarrow \infty$. Then, by the same way as in the proof of Theorem 1 it is possible to conclude that $\Delta \theta \rightarrow 0, Y_{1}-Y_{2} \rightarrow 0, \lambda_{1} \rightarrow 0$, $\lambda_{2} \rightarrow 0$ as $t \rightarrow \infty$, which completes the proof of Theorem 2 .

It is not so difficult to show that if one finger is of 2 DOF and the other is of 3 DOF (see Fig.5) and both finger-tip characteristics are the same in the sense that $r_{1}=r_{2}$ and $f_{1}(\Delta x)=f_{2}(\Delta x)$ for all $\Delta x \geq 0$ then it is possible to find two separate conjugate power pairs $u_{x i}$ and $u_{y i}$ together with velocity vector $\dot{q}_{i}$ posterior to $u_{f i}+u_{\theta i}$. Then, the principle of superposition leads to design of the feedback control input $u_{i}=u_{f i}+u_{\theta i}+u_{x i}+u_{y i}, i=1,2$, that realizes the stable grasping and desired posture $\left(\theta=\theta_{d}\right)$ and position $\left(x=x_{d}\right.$ and $\left.y=y_{d}\right)$ of the object (see Fig.2).

The principle of linear superposition suggests that learning the overall skill can be decomposed to learning each of resolved motions step by step in a manner as described by Fig.2. Then, complexity in learning the skill can be reduced from $N_{f} \times N_{\theta} \times N_{x} \times N_{y}$ to $N_{f}+N_{\theta}+N_{x}+N_{y}$ if $N_{f}$ (as well as $N_{\theta}, N_{x}, N_{y}$ ) signifies the number of exercises needed to acquire the control input signal $u_{f_{i}}(i=1,2)$ (as well as $u_{\theta_{i}}, u_{x_{i}}, u_{y_{i}}(i=$ $1,2))$. It can be interpreted that in the case of human learning some geometrical information on the Jacobian vectors appearing in eq.(20), that is, vectors $\alpha_{i}(1,1)^{\mathrm{T}}$ with $\alpha_{i}=r_{i} /\left(r_{1}+r_{2}\right)$ and $J_{0 i}^{\mathrm{T}}(\cos \theta,-\sin \theta)^{\mathrm{T}}$ must be acquired through the repeated practices, which in the sequel must be memorized as motor programs in a long-term memory in the brain. Computer simulation results show the effectiveness of the proposed design scheme for a class of skilled motions, which will be reported in our separate papers [7] [8].

5. Conclusions. This paper has shown that in both human and robot learning two physical principles called "principle of superposition" and "unique stationary resolution of position state variables" play an important role in learning a skilled motion 
such as pinching an object stably and regulating it at desired posture and position. This observation is obtained by strict analysis of nonlinear dynamiks of a mechanical robot model consisting of a set of two multi-degrees of freedom robot fingers with soft tips, which are derived on the basis of Euler-Lagrange's formalism. The two proposed principles must be one of such physical principles commonly underlying mechanical and living things as Shannon predicted in 1983 (see page XVII in [1])

\section{REFERENCES}

[1] N. J. A. Sloane And A. D. Wyner(eds.), Claude Elwood Shannon Collected Papers, IEEE Press, 1993.

[2] C. E. Shannon, Programming a computer for playing chess, first presented at the IRE Convention, March 9, 1949 and also reproduced in [1], pp. 637-645.

[3] S. Arimoto, P. T. A. NGuyen, H.-Y. Han, And Z. Doulgeri, Dynamics and control of a set of dual fingers with soft tips, Robotica, 18:1(2000), pp.71-80.

[4] S. Arimoto, K. Tahara, M. Yamaguchi, P. T. A. NguYen, and H.-Y. Han, Principle of Superposition for Controlling Pinch Motions by Means of Robot Fingers with Soft Tips, Preprints of IFAC Symp. on Robot Control (SYROCO 2000), Vienna, Austria, September 2000.

[5] J. P. LaSAlLE, Some extensions of Lyapunov's second method, IRE Trans. on Circuit Theory, $7(1960)$, pp. 520-527.

[6] V. Nguyen, Constructing stable grasps, International Journal of Robotics Research, 8:1(1989), pp. 26-37.

[7] P. T. A. NGuyen And S. ARimoto, Computer simulation of dynamics of dual fingers with softtips grasping an object, Proc. of the 2000 Japan-USA Symp. on Flexible Automation, Ann Arbor, Michigan, July 2000.

[8] P. T. A. NGUyen, S. ARimoto, AND H.-Y. Han, Computer simulation of controlled motion of dual fingers with soft-tips grasping and manipulating an object, submitted to Advanced Robotics, 2000.

Appendix A. It is necessary to prove the equality

$$
\begin{gathered}
\left(\dot{x}_{01}-\dot{x}_{02}\right) \sin \theta+\left(\dot{y}_{01}-\dot{y}_{02}\right) \cos \theta-r_{1}\left(\dot{q}_{11}+\dot{q}_{12}\right)+r_{2}\left(\dot{q}_{21}+\dot{q}_{22}\right)= \\
\left(l-\Delta x_{1}-\Delta x_{2}\right) \dot{\theta}
\end{gathered}
$$

To do this, note that two constraint eqs.(3) and (4) lead to

$$
\begin{aligned}
& \left(x_{1}-x_{2}\right)+l \cos \theta-\left(Y_{1}-Y_{2}\right) \sin \theta=0 \\
& \left(y_{1}-y_{2}\right)-l \sin \theta-\left(Y_{1}-Y_{2}\right) \cos \theta=0
\end{aligned}
$$

which gives rise to

$$
\left(x_{1}-x_{2}\right) \sin \theta+\left(y_{1}-y_{2}\right) \cos \theta=Y_{1}-Y_{2}
$$

Differentiating this in $t$ yields

$$
\begin{gathered}
\left(\dot{x}_{1}-\dot{x}_{2}\right) \sin \theta+\left(\dot{y}_{1}-\dot{y}_{2}\right) \cos \theta= \\
\dot{Y}_{1}-\dot{Y}_{2}-\left\{\left(x_{1}-x_{2}\right) \cos \theta-\left(y_{1}-y_{2}\right) \sin \theta\right\} \dot{\theta}
\end{gathered}
$$

Since the content of brackett \{\} of eq.(A-5) is equivalent to $l$ as easily calculated from eqs.(A-2) and (A-3), (A-5) can be rewritten, by referring to eqs.(1) and (2), in the form

$$
\begin{gathered}
\left(\dot{x}_{1}-\dot{x}_{2}\right) \sin \theta+\left(\dot{y}_{1}-\dot{y}_{2}\right) \cos \theta= \\
-\left(r_{1}+r_{2}-l\right) \dot{\theta}+r_{1}\left(\dot{q}_{11}+\dot{q}_{12}\right)-r_{2}\left(\dot{q}_{21}+\dot{q}_{22}\right)
\end{gathered}
$$


On the other hand, it is easy to see that

$$
\begin{aligned}
x_{i} & =x_{0 i}-(-1)^{i}\left(r_{i}-\Delta x_{i}\right) \cos \theta \\
y_{i} & =y_{0 i}+(-1)^{i}\left(r_{i}-\Delta x_{i}\right) \sin \theta
\end{aligned}
$$

from which it follows that

$$
\begin{gathered}
\left(\dot{x}_{1}-\dot{x}_{2}\right) \sin \theta+\left(\dot{y}_{1}-\dot{y}_{2}\right) \cos \theta= \\
\left(\dot{x}_{01}-\dot{x}_{02}\right) \sin \theta+\left(\dot{y}_{01}-\dot{y}_{02}\right) \cos \theta-\left(r_{1}+r_{2}-\Delta x_{1}-\Delta x_{2}\right) \dot{\theta}
\end{gathered}
$$

Substituting this into the left hand side of eq.(A-6) yields the equality (A-1).

Appendix B. We prove that $\lambda_{1}$ and $\lambda_{2}$ can be expressed in terms of state variables by substituting eqs.(1) and (2) of geometric constraints together with their first and second derivatives in $t$ into eqs.(23) to (26). In fact, let us denote

$$
S_{i}=Y_{i}-c_{i}+r_{i} \phi_{i}, \quad i=1,2
$$

and multiply eqs. (23) with $\Delta u_{i}=0$ by $\left(\partial S_{i} / \partial q_{i}\right)^{\mathrm{T}} H_{i}^{-1}\left(q_{i}\right)$ for $i=1,2$ and eqs.(17) by $\left(\partial S_{i} / \partial z\right)^{\mathrm{T}} H^{-1}$ for $i=1,2$. Then it follows that

$$
\begin{aligned}
& \left(\frac{\partial S_{i}}{\partial q_{i}}\right)^{\mathrm{T}} \ddot{q}_{i}+\lambda_{i} s_{i}^{\mathrm{T}} H_{i}^{-1}\left(q_{i}\right) s_{i}+g_{i}=0 \quad i=1,2 \\
& \left(\frac{\partial S_{i}}{\partial z}\right)^{\mathrm{T}} \ddot{z}+\sum_{j=1,2} \lambda_{j} p_{i}^{\mathrm{T}} H^{-1} p_{j}+h_{i}=0 \quad i=1,2
\end{aligned}
$$

where

$$
\begin{aligned}
& s_{i}=\frac{\partial S_{i}}{\partial q_{i}}=r_{i}\left(\begin{array}{c}
1 \\
1
\end{array}\right)-J_{0 i}^{\mathrm{T}}\left(\begin{array}{c}
\sin \theta \\
\cos \theta
\end{array}\right) \\
& p_{i}=\frac{\partial S_{i}}{\partial z}=\left(\begin{array}{c}
\sin \theta \\
\cos \theta \\
(-1)^{i}\left(\Delta x_{i}-\frac{1}{2} l\right)
\end{array}\right)
\end{aligned}
$$

where $g_{i}$ and $h_{i}$ denote corresponding remaining terms that are independent of $\lambda_{i}$ $(i=1,2), \ddot{q}_{i}(i=1,2)$, and $\ddot{z}$. Since $S_{i}=0$ for $i=1,2$, it follows that

$$
0=\ddot{S}_{i}=\left(\frac{\partial S_{i}}{\partial q_{i}}\right)^{\mathrm{T}} \ddot{q}_{i}+\left(\frac{\partial S_{i}}{\partial z}\right)^{\mathrm{T}} \ddot{z}+\left\{\frac{\mathrm{d}}{\mathrm{d} t}\left(\frac{\partial S_{i}}{\partial q_{i}}\right)^{\mathrm{T}}\right\} \dot{q}_{i}+\left\{\frac{\mathrm{d}}{\mathrm{d} t}\left(\frac{\partial S_{i}}{\partial q_{i}}\right)^{\mathrm{T}}\right\} \dot{z}
$$

Then, from eqs.(B-3), (B-4), and (B-7) it follows that

$$
\begin{array}{r}
\left(\alpha_{1}+\beta_{1}\right) \lambda_{1}+\gamma \lambda_{2}=g_{1}^{\prime} \\
\gamma \lambda_{1}+\left(\alpha_{2}+\beta_{2}\right) \lambda_{2}=g_{2}^{\prime}
\end{array}
$$

where

$$
\alpha_{i}=s_{i}^{\mathrm{T}} H_{i}^{-1} s_{i}, \beta_{i}=p_{i} H^{-1} p_{i}, \gamma=p_{1} H^{-1} p_{2}
$$

Since $\alpha_{i}>0, \beta_{i}>0$, and $\gamma^{2}=\beta_{1} \beta_{2}$, it is easy to check that eqs.(B-7) and (B-8) in $\lambda_{1}$ and $\lambda_{2}$ can be uniquely solved. Thus, it is proved that $\lambda_{1}$ and $\lambda_{2}$ can be expressed in terms of only state variables. 